H. Yin and Q. Qiu

Nagoya Math. J.

Vol. 154 (1999), 157-169

\title{
THE BLOWUP OF SOLUTIONS FOR 3-D AXISYMMETRIC COMPRESSIBLE EULER EQUATIONS
}

\author{
HUICHENG YIN AND QINGJIU QIU
}

\begin{abstract}
In this paper, for three dimensional compressible Euler equations with small perturbed initial data which are axisymmetric, we prove that the classical solutions have to blow up in finite time and give a complete asymptotic expansion of lifespan.
\end{abstract}

\section{$\S 1$. Introduction}

There are many results on the lifespan of classical solutions for nonlinear wave equations with small initial data ([1], [2] etc.). However, few papers have treated the problem of the lifespan of solutions for higher dimensional compressible Euler equations. In fact, it is very difficult to determine whether the smooth solutions of compressible Euler equations blow up or not. In [3], T. Sideris gave an upper bound for the lifespan of three dimensional Euler equations under appropriate conditions. In the paper, we will discuss 3-D problem with spherically symmetric initial data. Generally speaking, the spherically symmetric initial data don't satisfy the conditions in [3], so we can't give the upper bound of lifespan in light of the result in [3]. For the 2-D isentropic Euler equations with rotationally invariant data which are a perturbation of size $\varepsilon$ of a rest state, S. Alinhac [4] has established the lifespan of solution. For the general irrotational initial data (not spherically symmetric) which is a small perturbation, we can also determine the lifespan of classical solutions. This will be given in another paper.

Consider the following initial data problem for 3-D compressible Euler

Received March 26, 1997. 
equations:

$$
\left\{\begin{array}{l}
\partial_{t} \rho+\operatorname{div}(\rho v)=0 \\
\partial_{t} v+(v \nabla) v=-\frac{\nabla p(\rho)}{\rho}=-\frac{c^{2}(\rho)}{\rho} \nabla \rho \\
\left.\rho\right|_{t=0}=\bar{\rho}+\varepsilon \rho_{0}(x),\left.\quad v\right|_{t=0}=\varepsilon v_{0}(x)
\end{array}\right.
$$

where $\bar{\rho}>0$ is a constant, $c^{2}(\rho)=d p / d \rho>0, p(\rho) \in C^{\infty}$ for $\rho>0, \varepsilon>0$ sufficiently small, $v=\left(v_{1}, v_{2}, v_{3}\right), x=\left(x_{1}, x_{2}, x_{3}\right), \rho_{0}(x), v_{0}(x) \in C^{\infty}\left(R^{3}\right)$ and have compact supports in $|x| \leq R_{0}$. Moreover we assume that $v_{0}(x)=$ $v_{1}^{0}(x) x$, where $v_{1}^{0}$ is a smooth function in $R^{3}$, and $v_{1}^{0}(x), \rho_{0}(x)$ depend only on $r, r=\sqrt{x_{1}^{2}+x_{2}^{2}+x_{3}^{2}}$.

MAIN TheOREM. Under the above assumptions, (1) has a $C^{\infty}$ solution for $0 \leq t<T_{\varepsilon}$, where

$$
\begin{aligned}
\lim _{\varepsilon \rightarrow 0} & \varepsilon \ln T_{\varepsilon}=\tau_{0} \\
& =-\frac{2 \bar{c}}{\left(\bar{\rho} c^{\prime}(\bar{\rho})+\bar{c}\right) \min _{|q| \leq R_{0}}\left[q^{2} \partial_{q} v_{1}^{0}(q)+3 q v_{1}^{0}(q)+\frac{\bar{c}}{\bar{\rho}}\left(q \partial_{q} \rho_{0}(q)+\rho_{0}(q)\right)\right]}
\end{aligned}
$$

and $\bar{c}=c(\bar{\rho}), T_{\varepsilon}$ denotes the lifespan of smooth solution.

Remark. It is easy to know $\min _{|q| \leq R_{0}}\left[q^{2} \partial_{q} v_{1}^{0}(q)+3 q v_{1}^{0}(q)+\bar{c}\left(q \partial_{q} \rho_{0}(q)+\right.\right.$ $\left.\left.\rho_{0}(q)\right) / \bar{\rho}\right]<0$ unless $v_{1}^{0} \equiv 0, \rho_{0} \equiv 0$. Moreover $\bar{\rho} c^{\prime}(\bar{\rho})+\bar{c}>0$ is known (see [5]).

For proving Theorem, we note that the rotations of $v$ are zero, then (1) will be reduced into a nonlinear wave equation. As in [2], by constructing an approximate solution and considering the difference of exact solution and approximate solution, we easily get the lower bound of lifespan. On the other hand, the spherical symmetricity of solution makes us to change (1) into a $2 \times 2$ system equation in two variables $(r, t)$. Hence by using the properties of above approximate solution and imitating the proof in [4], [6], [7], we may obtain the estimate of upper bound for the lifespan. Theorem asserts that the solution of (1) blows up in finite time unless $v_{0} \equiv 0, \rho_{0} \equiv 0$ in spite of any small $\varepsilon$.

\section{$\S 2$. The lower bound of lifespan $T_{\varepsilon}$}

Under the assumptions of Theorem, we know the solution of (1) has such a form in $t<T_{\varepsilon}: \rho(x, t)=\rho(r, t), v(x, t)=\tilde{v}(r, t) x$, where $\tilde{v}(r, t)$ 
is a smooth function of $(r, t)$. Because of $\operatorname{rot} v(x, t)=0$, there exists a function $\omega(x, t) \in C^{\infty}$, such that $v(x, t)=\nabla \omega(x, t)$, moreover $\omega(x, t)$ has compact support in $x$ (by the finite propagation speed we know $v$ has compact support), and depends only on $r$. If we denote $\omega(r, t)=\omega(x, t)$, then $\partial_{r} \omega(r, t)=r \tilde{v}(r, t)$. Substituing $v(x, t)=\nabla \omega$ into the second equation in $(1)$, then we have:

$$
\partial_{t} \nabla \omega+\nabla\left(\frac{1}{2}|\nabla \omega|^{2}\right)=-\nabla h(\rho)
$$

where $h^{\prime}(\rho)=c^{2}(\rho) / \rho$, and $h(\bar{\rho})=0$. Hence $\partial_{t} \omega+|\nabla \omega|^{2} / 2=-h(\rho)$.

Noting $h^{\prime}(\rho)>0$, and by implicit function theorem, we know that

$$
\rho=h^{-1}\left(-\left(\partial_{t} \omega+\frac{1}{2}|\nabla \omega|^{2}\right)\right), \quad \bar{\rho}=h^{-1}(0)
$$

Therefore, from the first eqution in (1), it turns out

$$
\begin{aligned}
\partial_{t}^{2} \omega+ & 2 \sum_{k=1}^{3} \partial_{k} \omega \partial_{t} \partial_{k} \omega-\frac{h^{-1}\left(-\left(\partial_{t} \omega+\frac{1}{2}|\nabla \omega|^{2}\right)\right)}{\left(h^{-1}\right)^{\prime}\left(-\left(\partial_{t} \omega+\frac{1}{2}|\nabla \omega|^{2}\right)\right)} \Delta \omega \\
& +\sum_{i, k=1}^{3} \partial_{i} \omega \partial_{k} \omega \partial_{i} \partial_{k} \omega=0
\end{aligned}
$$

Now we determine the initial data $\left.\omega\right|_{t=0}$ and $\left.\partial_{t} \omega\right|_{t=0}$.

Obviously, $\left.\omega\right|_{t=0}=\varepsilon \int_{R_{0}}^{r} s v_{1}^{0}(s) d s$.

Since $\left.\partial_{t} \nabla \omega\right|_{t=0}+\left.\nabla\left(|\nabla \omega|^{2}\right)\right|_{t=0} / 2=-\left.c^{2}(\rho) \nabla \rho\right|_{t=0} / \rho$, then we have

$$
\begin{aligned}
& \left.\partial_{t} \partial_{r} \omega\right|_{t=0}+\frac{1}{2} \varepsilon^{2} \partial_{r}\left[r^{2}\left(v_{1}^{0}(r)\right)^{2}\right] \\
& =-\varepsilon \frac{\bar{c}^{2}}{\bar{\rho}} \partial_{r} \rho_{0}-\left.\varepsilon^{2} \int_{0}^{1}\left(\frac{c^{2}(\rho)}{\rho}\right)^{\prime}\right|_{\rho=\bar{\rho}+\theta \varepsilon \rho_{0}} d \theta \rho_{0} \partial_{r} \rho_{0} .
\end{aligned}
$$

Set

$$
g(x, \varepsilon)=-\int_{R_{0}}^{r}\left[\left.\int_{0}^{1}\left(\frac{c^{2}(\rho)}{\rho}\right)^{\prime}\right|_{\rho=\bar{\rho}+\theta \varepsilon \rho_{0}(s)} d \theta\right] \rho_{0}(s) \partial_{s} \rho_{0}(s) d s-\frac{1}{2} r^{2}\left(v_{1}^{0}(r)\right)^{2},
$$

then $g(x, \varepsilon)$ is smooth in $x, \varepsilon$ and has compact support in $r \leq R_{0}$. So $\left.\partial_{t} \omega\right|_{t=0}=-\varepsilon \bar{c}^{2} \rho_{0} / \bar{\rho}+\varepsilon^{2} g(x, \varepsilon)$. 
Then for considering the lower bound of lifespan for (1), we only discuss the lower bound of lifespan for the following problem:

(2)

$$
\left\{\begin{aligned}
\partial_{t}^{2} \omega+ & 2 \sum_{k=1}^{3} \partial_{k} \omega \partial_{t} \partial_{k} \omega-\frac{h^{-1}\left(-\left(\partial_{t} \omega+\frac{1}{2}|\nabla \omega|^{2}\right)\right)}{\left(h^{-1}\right)^{\prime}\left(-\left(\partial_{t} \omega+\frac{1}{2}|\nabla \omega|^{2}\right)\right)} \Delta \omega \\
& +\sum_{i, k=1}^{3} \partial_{i} \omega \partial_{k} \omega \partial_{i} \partial_{k} \omega=0 \\
\left.\omega\right|_{t=0}= & \varepsilon \int_{R_{0}}^{r} s v_{1}^{0}(s) d s \\
\left.\partial_{t} \omega\right|_{t=0}= & -\varepsilon \frac{\bar{c}^{2}}{\bar{\rho}} \rho_{0}+\varepsilon^{2} g(x, \varepsilon) .
\end{aligned}\right.
$$

It is easy to know $\frac{h^{-1}\left(-\left(\partial_{t} \omega+|\nabla \omega|^{2} / 2\right)\right)}{\left(h^{-1}\right)\left(-\left(\partial_{t} \omega+|\nabla \omega|^{2} / 2\right)\right)}=\bar{c}^{2}-2 \bar{\rho} c^{\prime}(\bar{\rho}) \partial_{t} \omega / \bar{c}+O\left(\left|\nabla_{x, t} \omega\right|^{2}\right)$.

we set

$$
\left\{\begin{array}{l}
\partial_{t}^{2} \omega_{0}-\bar{c}^{2} \Delta \omega_{0}=0 \\
\left.\omega_{0}\right|_{t=0}=\int_{R_{0}}^{r} s v_{1}^{0}(s) d s \\
\left.\partial_{t} \omega_{0}\right|_{t=0}=-\frac{\bar{c}^{2}}{\bar{\rho}} \rho_{0}
\end{array}\right.
$$

and

$$
\left\{\begin{array}{l}
\frac{\partial U(s, q)}{\partial s}=-\frac{\bar{\rho} c^{\prime}(\bar{\rho})+\bar{c}}{2 \bar{c}^{2}}\left(\frac{\partial U(s, q)}{\partial q}\right)^{2} \\
U(0, q)=F_{0}(q) .
\end{array}\right.
$$

Where $F_{0}(q)$ is the Friedlander radiation field of (3). By [2, (6.2.12)], we have

$$
\begin{aligned}
\frac{\partial F_{0}(q)}{\partial q} & =\frac{1}{2}\left[\bar{c} \frac{d}{d q}\left(q \int_{R_{0}}^{q} s v_{1}^{0}(s) d s\right)+q \frac{\bar{c}^{2}}{\bar{\rho}} \rho_{0}(q)\right] \\
& =\frac{\bar{c}}{2}\left(\int_{R_{0}}^{q} s v_{1}^{0}(s) d s+q^{2} v_{1}^{0}(q)+\frac{\bar{c}}{\bar{\rho}} q \rho_{0}(q)\right) .
\end{aligned}
$$

Set $\omega_{a}=\varepsilon\left[\chi(\varepsilon t) \omega_{0}+(1-\chi(\varepsilon t)) r^{-1} U(\varepsilon \ln (\varepsilon t), r-\bar{c} t)\right]$, where $\chi \in C^{\infty}(R)$ decreases, equals to 1 in $(-\infty, 1)$, and equals to 0 in $(2, \infty)$. Then we have the following conclusion:

Lemma 1. For sufficiently small $\varepsilon>0$, and $\varepsilon \ln T<b<\tau_{0}$, then 
(i) (2) has a $C^{\infty}$ solution for $0<t \leq T$.

(ii) $\left|\partial_{x, t}^{\beta}\left(\omega-\omega_{a}\right)\right| \leq \frac{C_{\beta, b} \varepsilon^{2} \ln (1 / \varepsilon)}{1+t},|\beta| \leq 2$.

(iii) $\left|\partial_{x, t}^{\beta} \nabla_{x, t} \omega_{a}\right| \leq \frac{C_{\beta, b} \varepsilon}{1+t},|\beta| \geq 0$.

Proof. (i) Noting $\partial^{2} F_{0}(q) / \partial q^{2}=\bar{c}\left[q^{2} \partial_{q} v_{1}^{0}(q)+3 q v_{1}^{0}(q)+\bar{c} q \partial_{q} \rho_{0}(q) / \bar{\rho}+\right.$ $\left.\bar{c} \rho_{0}(q) / \bar{\rho}\right] / 2$, as in [2, Theorem 6.5.7], we know (i) holds.

(ii) By [2, Lemma 6.5.6] and S.Klainerman's inequality in [8], we easily know (ii) holds.

(iii) Its proof is similar to that in [2, Lemma 6.5.5].

From Lemma 1 (i), it is easy to know that $\lim _{\varepsilon \rightarrow 0} \varepsilon \ln T_{\varepsilon} \geq \tau_{0}$.

Remark. We state that $\min _{|q| \leq M}\left[q^{2} \partial_{q} v_{1}^{0}(q)+3 q v_{1}^{0}(q)+\bar{c} q \partial_{q} \rho_{0}(q) / \bar{\rho}\right]<0$ unless $v_{0} \equiv 0$ and $\rho_{0} \equiv 0$. In fact, if $\min _{|q| \leq M}\left[q^{2} \partial_{q} v_{1}^{0}(q)+3 q v_{1}^{0}(q)+\right.$ $\left.\bar{c} q \partial_{q} \rho_{0}(q) / \bar{\rho}\right] \geq 0$, then $\partial^{2} F_{0}(q) / \partial q^{2} \geq 0$. Because $F_{0}(q)$ has compact support in $|q| \leq M$, then $\partial F_{0}(q) / \partial q \equiv 0$, hence $F_{0}(q) \equiv 0$. By [2, Theorem $6.2 .2]$, we have $v_{1}^{0} \equiv 0, \rho_{0} \equiv 0$.

\section{$\S 3$. The upper bound of lifespan $T_{\varepsilon}$}

Assume that $\omega_{a}, b$ are defined as above, when $\varepsilon \ln T \leq b$, we set $\rho_{a}=$ $h^{-1}\left(-\left(\partial_{t} \omega_{a}+\left|\nabla \omega_{a}\right|^{2} / 2\right)\right)$. Since

$$
\begin{aligned}
\rho-\rho_{a}=- & \int_{0}^{1}\left(h^{-1}\right)^{\prime}\left(-\theta\left(\partial_{t} u+\frac{1}{2}|\nabla u|^{2}\right)-(1-\theta)\left(\partial_{t} u_{a}+\frac{1}{2}\left|\nabla u_{a}\right|^{2}\right)\right) d \theta \\
& \times\left[\partial_{t}\left(u-u_{a}\right)+\frac{1}{2}\left(\nabla u+\nabla u_{a}\right) \nabla\left(u-u_{a}\right)\right]
\end{aligned}
$$

then by lemma 1 , then we obtain

$$
\left|\partial_{x, t}^{\beta}\left(\rho-\rho_{a}\right)\right| \leq \frac{C_{\beta, b} \varepsilon^{2} \ln (1 / \varepsilon)}{1+t}, \quad|\beta| \leq 2
$$

and

$$
\left|\partial_{x, t}^{\beta}\left(\rho_{a}-\bar{\rho}\right)\right| \leq \frac{C_{\beta, b} \varepsilon}{1+t}, \quad|\beta| \geq 0
$$

Set $\alpha(r, t)=\partial_{r} \omega(r, t), \alpha_{a}(r, t)=\partial_{r} \omega_{a}(r, t)$. For the smooth solution of $(1)$, we have 
LEMMA 2. (a) $\alpha, \rho$ satisfy the following system:

$$
\left\{\begin{array}{l}
\partial_{t} \rho+\alpha \partial_{r} \rho+\rho\left(\partial_{r} \alpha+\frac{2}{r} \alpha\right)=0 \\
\partial_{t} \alpha+\alpha \partial_{r} \alpha+\frac{c^{2}(\rho)}{\rho} \partial_{r} \rho=0
\end{array}\right.
$$

(b) Set $\rho=\bar{\rho}+A(r, t) / r, \alpha=B(r, t) / r, Z_{1}=\left(\partial_{r} A / \rho+\partial_{r} B / c\right) / 2$, $Z_{2}=\left(-\partial_{r} A / \rho+\partial_{r} B / c\right) / 2$, then $\left(Z_{1}, Z_{2}\right)$ satisfy the following system:

$$
\left\{\begin{array}{l}
\partial_{t} Z_{1}+(\alpha+c) \partial_{r} Z_{1}=Q_{1} \\
\partial_{t} Z_{2}+(\alpha-c) \partial_{r} Z_{2}=Q_{2}
\end{array}\right.
$$

where

$$
\begin{aligned}
Q_{1} & =-\frac{Z_{1}^{2}}{r}\left(c+c^{\prime} \rho\right)+\frac{Z_{1} Z_{2}}{r}\left(3 c^{\prime} \rho+\frac{B}{r}\right)+\frac{Z_{2}^{2}}{r}\left(c-\frac{B}{r}\right) \\
+ & \frac{Z_{1}}{r^{2}}\left[3 B+\frac{c A}{\rho}+\frac{c^{\prime} A}{2}+\frac{c^{\prime} B \rho}{2 c}+\left(\frac{c^{2}(\rho)}{\rho}\right)^{\prime} \frac{\rho A}{c}-\frac{B \bar{\rho}}{2 \rho}-\frac{A B}{2 \rho r}+\frac{c r}{2 \rho}(\rho-\bar{\rho})\right] \\
& +\frac{Z_{2}}{r^{2}}\left[B-\frac{c A}{\rho}-\left(\frac{c^{2}(\rho)}{\rho}\right)^{\prime} \frac{\rho A}{c}+\frac{c^{\prime} B \rho}{2 c}+\frac{c A}{\rho}+\frac{c^{\prime} A}{2}+\frac{B \bar{\rho}}{2 \rho}-\frac{c B}{2 \rho r}-\frac{c r}{2}-\frac{\bar{\rho} c r}{2 \rho}\right] \\
& +\frac{1}{r^{2}}\left(-\frac{c A}{2 \rho}+B-\frac{\bar{\rho} B}{2 \rho}\right)+\frac{1}{r^{3}}\left[-\frac{B^{2}}{c}-\left(\frac{c^{2}(\rho)}{\rho}\right)^{\prime} \frac{A^{2}}{2 c}-\frac{3 A B}{2 \rho}+\frac{\rho c B}{2 \rho^{2}}\right] \\
+ & \frac{A B c}{2 \rho^{2} r^{4}}, \\
Q_{2} & =\frac{Z_{1} Z_{2}}{r}\left(3 c^{\prime} \rho-2 A-\frac{B}{r}\right)+\frac{Z_{2}^{2}}{r}\left(-2 A+\frac{B}{r}-c^{\prime} \rho\right) \\
& +\frac{Z_{1}}{r^{2}}\left[\left(\frac{c^{2}(\rho)}{\rho}\right)^{\prime} \frac{\rho A}{c}+\frac{c r}{2}+\frac{c^{\prime} B \rho}{2 c}-\frac{c^{\prime} A}{2}+\frac{B \bar{\rho}}{2 \rho}+\frac{\bar{\rho}}{2 \rho} r+\frac{B c}{2 \rho r}\right] \\
& +\frac{Z_{2}}{r^{2}}\left[2 B-\frac{c r}{2}-\left(\frac{c^{2}(\rho)}{\rho}\right)^{\prime} \frac{\rho A}{c}-\frac{c A}{\rho}+\frac{c^{\prime} B \rho}{2 c}-\frac{c^{\prime} A}{2}-\frac{B \bar{\rho}}{2 \rho}+\frac{\bar{\rho} c}{2 \rho} r+\frac{B c}{2 \rho r}\right] \\
& +\frac{1}{r^{2}}\left(-\frac{c A}{2 \rho}-B+\frac{\bar{\rho} B}{2 \rho}\right)+\frac{1}{r^{3}}\left[-\frac{B^{2}}{c}-\left(\frac{c^{2}(\rho)}{\rho}\right)^{\prime} \frac{A^{2}}{2 c}+\frac{3 A B}{2 \rho}-\frac{\bar{\rho} B c}{2 \rho^{2}}\right] \\
& -\frac{A B c}{2 \rho^{2} r^{4}} .
\end{aligned}
$$

Remark. It is very important to appear the factor $\operatorname{cr}(\rho-\bar{\rho}) / 2 \rho$ in the coefficients of $Z_{1} / r^{2}$ in $Q_{1}$, or we can't give the upper bound of lifespan $T_{\varepsilon}$ of solution to (1). 
Proof. (a) It can be verified directly, we omit it.

(b) Since $Z_{1}+Z_{2}=\partial_{r} B / c=\left(\alpha+r \partial_{r} \alpha\right) / c, Z_{1}-Z_{2}=\left(r \partial_{r} \rho+\rho-\bar{\rho}\right) / \rho$, thus

$$
\begin{aligned}
& \partial_{r} \alpha=\frac{c\left(Z_{1}+Z_{2}\right)}{r}-\frac{B}{r^{2}}, \quad \partial_{r} \rho=\frac{\rho\left(Z_{1}-Z_{2}\right)}{r}-\frac{A}{r^{2}}, \\
& \partial_{r}^{2} \alpha=\frac{c \partial_{r}\left(Z_{1}+Z_{2}\right)}{r}+\frac{c^{\prime} \rho\left(Z_{1}^{2}-Z_{2}^{2}\right)}{r^{2}}-\frac{A c^{\prime}\left(Z_{1}+Z_{2}\right)}{r^{3}}-\frac{2 c\left(Z_{1}+Z_{2}\right)}{r^{2}}+\frac{2 B}{r^{3}} \\
& \partial_{r}^{2} \rho=\frac{\rho \partial_{r}\left(Z_{1}-Z_{2}\right)}{r}+\frac{\rho\left(Z_{1}-Z_{2}\right)^{2}}{r^{2}}-\frac{A\left(Z_{1}-Z_{2}\right)}{r^{3}}-\frac{2 \rho\left(Z_{1}-Z_{2}\right)}{r^{2}}+\frac{2 A}{r^{3}} .
\end{aligned}
$$

then

$$
\begin{aligned}
& \partial_{t}\left(Z_{1}+Z_{2}\right)=-\frac{B}{r} \partial_{r}\left(Z_{1}+Z_{2}\right)-c \partial_{r}\left(Z_{1}-Z_{2}\right)-\frac{c\left(Z_{1}-Z_{2}\right)}{r} \\
& -\frac{c}{r}\left(Z_{1}+Z_{2}\right)^{2}+\frac{B}{r^{2}}\left(Z_{1}+Z_{2}\right)-\frac{2 B^{2}}{c r^{3}}+\frac{2 B\left(Z_{1}+Z_{2}\right)}{r^{2}} \\
& -\left(\frac{c^{2}(\rho)}{\rho}\right)^{\prime}\left[\frac{\rho^{2}\left(Z_{1}-Z_{2}\right)^{2}}{c r}-\frac{2 \rho A\left(Z_{1}-Z_{2}\right)}{c r^{2}}+\frac{A^{2}}{c r^{3}}\right] \\
& -\frac{c\left(Z_{1}-Z_{2}\right)^{2}}{r}+\frac{c A\left(Z_{1}-Z_{2}\right)}{\rho r^{2}}+\frac{2 c\left(Z_{1}-Z_{2}\right)}{r}-\frac{c A}{\rho r^{2}} \\
& +\frac{\rho c^{\prime}\left(Z_{1}+Z_{2}\right)^{2}}{r}+\frac{c^{\prime} \rho B\left(Z_{1}+Z_{2}\right)}{c r^{2}}
\end{aligned}
$$

and

$$
\begin{gathered}
\partial_{t}\left(Z_{1}-Z_{2}\right)=-\frac{B}{r}\left(Z_{1}-Z_{2}\right)-c \partial_{r}\left(Z_{1}-Z_{2}\right)-\frac{2 c}{r}\left(Z_{1}^{2}-Z_{2}^{2}\right) \\
+\frac{2 c A\left(Z_{1}+Z_{2}\right)}{\rho r^{2}}-\frac{3 A B}{\rho r^{3}}-\frac{B\left(Z_{1}-Z_{2}\right)^{2}}{r^{2}}+\frac{2 B\left(Z_{1}-Z_{2}\right)}{r^{2}} \\
\quad-\frac{c^{\prime} \rho}{r}\left(Z_{1}^{2}-Z_{2}^{2}\right)+\frac{c^{\prime} A\left(Z_{1}+Z_{2}\right)}{r^{2}}+\frac{2 B}{r^{2}}-\frac{B \bar{\rho}\left(Z_{1}-Z_{2}\right)}{\rho r^{2}} \\
+\frac{\bar{\rho} B c}{\rho^{2} r^{3}}-\frac{\bar{\rho} c\left(Z_{1}+Z_{2}\right)}{\rho r}-\frac{\bar{\rho} B}{\rho r^{2}}+\frac{B\left(z_{1}^{2}-Z_{2}^{2}\right)}{r^{2}}-\frac{B c\left(Z_{1}+Z_{2}\right)}{\rho r^{3}} \\
+\frac{c\left(Z_{1}+Z_{2}\right)^{2}}{r}+\frac{B\left(Z_{1}+Z_{2}\right)}{r^{2}}+\frac{A B c}{\rho^{2} r^{4}}-\frac{A c\left(Z_{1}+Z_{2}\right)}{\rho r^{2}} .
\end{gathered}
$$

From (8) and (9), it is easy to obtain (7). Hence Lemma 2 is proved.

Denote $\Gamma_{\lambda}^{ \pm}$as the integral curves of $\partial_{t} \pm(\alpha+c) \partial_{r}$ passed through $(\lambda, 0)$ in the plan $(r, t), D$ as the strip domain bounded by $\Gamma_{R_{0}}^{+}$and $\Gamma_{\sigma_{0}-1}^{+}$where 
$\sigma_{0}$ is taken such that $\left(q^{2} \partial_{q} v_{1}^{0}(q)+3 q v_{1}^{0}(q)+\bar{c} q \partial_{q} \rho_{0}(q) / \bar{\rho}+\left.\bar{c} \rho_{0}(q)\right|_{q=\sigma_{0}} / \bar{\rho}\right)$ is minimum. $D_{\varepsilon}=D \cap\{t \geq 1 / 2 \varepsilon\}$. As in [4], [6], [7], for any $T$ satisfying $1 / 2 \varepsilon \leq T \leq e^{b / \varepsilon}, b<\tau_{0}$, we define

$$
J(t)=\sup _{\substack{1 / 2 \varepsilon \leq s \leq t \\(r, s) \in D}} \int\left|Z_{1}(r, s)\right| d r, \quad M(t)=\sup _{\substack{1 / 2 \varepsilon \leq s \leq t \\(r, s) \in D}}(|A(r, s)|+|B(r, s)|),
$$

and $V(t)=\sup _{\substack{1 / 2 \varepsilon \leq s \leq t \\(r, s) \in D}} s\left|Z_{2}(r, s)\right|$

LEMMA 3. There exist some constants $J_{1}, M_{1}, V_{1}>0$, for sufficiently small $\varepsilon>0$ and any $t$ satisfying $1 / 2 \varepsilon \leq t \leq T \leq e^{b / \varepsilon}$, such that $J(t) \leq$ $J_{1} \varepsilon, M(t) \leq M_{1} \varepsilon, V(t) \leq V_{1} \varepsilon^{1 / 2}$. Moreover $r \geq \bar{c} t / 2$ in $D_{\varepsilon}$.

Proof. Firstly we verify the lemma for $1 / 2 \varepsilon \leq t \leq 1 / \varepsilon$.

Set $\rho=\rho_{a}+\dot{\rho}$. It turns out

$$
2 Z_{1}=r\left(\frac{\partial_{r} \rho_{a}+\partial_{r} \dot{\rho}}{\rho}+\frac{\partial_{r} \alpha}{c}\right)+\left(\frac{\rho_{a}-\bar{\rho}+\dot{\rho}}{\rho}+\frac{\alpha}{c}\right)
$$

and

$$
2 Z_{2}=r\left(-\frac{\partial_{r} \rho_{a}+\partial_{r} \dot{\rho}}{\rho}+\frac{\partial_{r} \alpha}{c}\right)+\left(-\frac{\rho_{a}-\bar{\rho}+\dot{\rho}}{\rho}+\frac{\alpha}{c}\right)
$$

Since

$$
\begin{aligned}
& t\left|2 Z_{2}-r\left(\frac{\partial_{r} \alpha_{a}}{c}-\frac{\partial_{r} \rho_{a}}{\rho}\right)\right| \\
& \quad \leq t\left|r\left(-\frac{\partial_{r} \dot{\rho}}{\rho}+\frac{\partial_{r} \alpha-\partial_{r} \alpha_{a}}{c}\right)+\left(-\frac{\rho_{a}-\bar{\rho}+\dot{\rho}}{\rho}+\frac{\alpha}{c}\right)\right| \\
& \quad \leq \operatorname{cte}\left(\varepsilon+\varepsilon \ln \frac{1}{\varepsilon}\right) .
\end{aligned}
$$

Noting that

$$
\frac{\partial_{r} \alpha_{a}}{c}-\frac{\partial_{r} \rho_{a}}{\rho}=\frac{(\rho-\bar{\rho}) \partial_{r} \alpha_{a}}{\rho c}-\frac{(c-\bar{c}) \partial_{r} \rho_{a}}{\rho c}+\frac{\bar{\rho} \partial_{r} \alpha_{a}-\bar{c} \partial_{r} \rho_{a}}{\rho c}
$$

and

$$
\begin{aligned}
\bar{\rho} \partial_{r} \alpha_{a}-\bar{c} \partial_{r} \rho_{a}=\frac{\bar{\rho}}{\bar{c}} & \varepsilon\left(\bar{c} \partial_{r}+\partial_{t}\right) \partial_{r}\left(\omega_{a}-\frac{F_{0}(r-\bar{c} t)}{r}\right) \\
& +\varepsilon \bar{\rho}\left[\frac{2 F_{0}(r-\bar{c} t)}{r^{3}}-\frac{\partial_{q} F_{0}(r-\bar{c} t)}{r^{2}}\right] \\
+ & O\left(\left|\nabla_{x, t} \omega_{a}\right|^{2}\right)+O\left(\left|\nabla \omega_{a} \nabla \partial_{r} \omega_{a}\right|\right)
\end{aligned}
$$


Thus, by

$$
\left|\partial_{x, t}^{\beta}\left(\omega_{a}-\frac{F_{0}(r-\bar{c} t)}{r}\right)\right| \leq \frac{C_{\beta}}{(1+t)^{2}}, \quad|\beta| \geq 0,\left(r \geq \frac{\bar{c}}{2} t\right)
$$

we know

$$
t\left|Z_{2}\right| \leq \operatorname{tr}\left|\frac{\partial_{r} \alpha_{a}}{c}-\frac{\partial_{r} \rho_{a}}{\rho}\right|+\operatorname{cte}\left(\varepsilon+\varepsilon \ln \frac{1}{\varepsilon}\right) \leq c t e \varepsilon^{1 / 2}
$$

i.e.,

$$
V(t) \leq c t e \varepsilon^{1 / 2}
$$

On the other hand, owing to the width of $D$ is finite and $\left|Z_{1}\right| \leq$ ctee, then

$$
J(t) \leq \text { ctee, } \quad M(t)=\sup _{\substack{1 / 2 \varepsilon \leq s \leq t \\(r, s) \in D}}(|r \alpha|+|r(\rho-\bar{\rho})|) \leq \text { cte } \varepsilon .
$$

We choose $M_{1}=\left(2 \bar{c}^{2}+2 \bar{c} \bar{\rho}\right) J_{1}, V_{1}=J_{1}$, s.t. $J(1 / 2 \varepsilon) \leq J_{1} \varepsilon / 4, M(1 / 2 \varepsilon) \leq$ $M_{1} \varepsilon / 4$ and $V(1 / 2 \varepsilon) \leq J_{1} \varepsilon^{1 / 2} / 4$.

Now we verify the lemma as $1 / 2 \varepsilon \leq t \leq T^{\prime} \leq T$. For this aim, as in [4], we first claim that:

(i) On $\Gamma_{\lambda}^{+} \subset D$, then $|r-\bar{c} t-\lambda| \leq c t e$. In particular, for $t \geq 1 / 2 \varepsilon$ and $\varepsilon$ small enough, then $r \geq 3 \bar{c} t / 4$.

In fact, along $\Gamma_{\lambda}^{+} \subset D, d r-\bar{c} d t=(\alpha+c-\bar{c}) d t$, then

$$
\begin{aligned}
& |r-\bar{c} t-\lambda| \leq c t e \int_{0}^{t}|\alpha+c-\bar{c}| d t \\
& \quad \leq c t e \int_{0}^{t} \frac{\varepsilon}{1+t} d t \leq c t e \varepsilon \ln (1+t) \leq c t e .
\end{aligned}
$$

(ii) If $(r, t) \in \Gamma_{\mu}^{-} \cap D_{\varepsilon},\left(r^{\prime}, t^{\prime}\right) \in \Gamma_{\mu}^{-} \cap D_{\varepsilon}, t^{\prime} \leq t$, then $t-t^{\prime} \leq c t e$. In particular, $t^{\prime} \geq t / 2$ for sufficiently small $\varepsilon$.

In fact,

$$
\left|\frac{d}{d t}(r+\bar{c} t-\mu)\right| \leq \frac{c t e \varepsilon}{1+t}
$$

and

$$
\left|r(t)+\bar{c} t-\mu-\left(r\left(t^{\prime}\right)+\bar{c} t^{\prime}-\mu\right)\right| \leq c t e \varepsilon \ln \frac{1+t}{1+t^{\prime}} \leq c t e .
$$

Then imitating the proof in [4], we know the statement (ii) holds. 
Choosing $\mu=-\alpha-c$, along the integtal curve in $D$, we write $d\left(Z_{1}(d r+\mu d t)\right)=\left[(\alpha+c+\mu) \partial_{r} Z_{1}+Z_{1} \partial_{r} \mu-Q_{1}\right] d r \wedge d t=\widetilde{Q} d r \wedge d t$ where

$$
\begin{aligned}
\widetilde{Q} & =-\frac{Z_{1} Z_{2}}{r}\left(c+2 c^{\prime} \rho+\frac{B}{r}\right)-\frac{Z_{2}^{2}}{r}\left(c-\frac{B}{r}\right) \\
& -\frac{Z_{1}}{r^{2}}\left[2 B+\frac{c A}{\rho}-\frac{c A}{2}+\frac{c^{\prime} \rho B}{2 c}+\left(\frac{c^{2}(\rho)}{\rho}\right)^{\prime} \frac{\rho A}{c}-\frac{B \bar{\rho}}{2 \rho}-\frac{A B}{2 \rho r}+\frac{c r}{2 \rho}(\rho-\bar{\rho})\right] \\
& -\frac{Z_{2}}{r^{2}}\left[B-\frac{c A}{\rho}-\left(\frac{c^{2}(\rho)}{\rho}\right)^{\prime} \frac{\rho A}{c}+\frac{c^{\prime} B \rho}{2 c}+\frac{c A}{\rho}+\frac{c A}{2}+\frac{B \bar{\rho}}{2 \rho}-\frac{B c}{2 \rho r}-\frac{c r}{2}-\frac{\bar{\rho} c r}{2 \rho}\right] \\
& -\frac{1}{r^{2}}\left(-\frac{c A}{2 \rho}+B-\frac{\bar{\rho} B}{2 \rho}\right)-\frac{1}{r^{3}}\left[-\frac{B^{2}}{c}-\left(\frac{c^{2}(\rho)}{\rho}\right)^{\prime} \frac{A^{2}}{2 c}-\frac{3 A B}{2 \rho}+\frac{\bar{\rho} B c}{2 \rho^{2}}\right] \\
& -\frac{c A B}{2 \rho^{2} r^{4}} .
\end{aligned}
$$

As in [4], we have

$$
\begin{aligned}
& \text { (i) } \int_{(r, t) \in D}\left|Z_{1}(r, t)\right| d r \leq J\left(\frac{1}{2 \varepsilon}\right)+\int_{\substack{1 / 2 \varepsilon \leq s \leq t \\
(r, s) \in D}}|\widetilde{Q}| d r d s \\
& \text { (ii) cte } \int_{\Gamma^{-}(x, t) \cap D_{\varepsilon}}\left|Z_{1}(r, t)\right| d r \leq J\left(\frac{1}{2 \varepsilon}\right)+\int_{\substack{1 / 2 \varepsilon \leq s \leq t \\
(r, s) \in D}}|\widetilde{Q}| d r d s
\end{aligned}
$$

where $\Gamma^{-}(x, t)$ is the integral curve of $\partial_{t}-(\alpha+c) \partial_{r}$ passed through $(x, t) \in$ $D_{\varepsilon}$.

In $D_{\varepsilon}$, for sufficiently small $\varepsilon$, we have

$$
\begin{aligned}
|\widetilde{Q}| \leq c t e & {\left[\left|Z_{1}\right|\left(\frac{V_{1} \varepsilon^{1 / 2}}{t^{2}}+\frac{M_{1} \varepsilon}{t^{2}}+\frac{M_{1}^{2} \varepsilon^{2}}{t^{3}}+\frac{M_{1} V_{1} \varepsilon^{3 / 2}}{t^{3}}+\frac{\varepsilon}{t^{2}}\right)\right.} \\
+ & \left.\frac{V_{1}^{2} \varepsilon}{t^{3}}\left(1+\frac{M_{1} \varepsilon}{t}\right)+\frac{V_{1} \varepsilon^{1 / 2}}{t^{3}}\left(M_{1} \varepsilon+t\right)+\frac{M_{1} \varepsilon}{t^{2}}+\frac{M_{1}^{2} \varepsilon^{2}}{t^{3}}+\frac{M_{1}^{2} \varepsilon^{2}}{t^{4}}\right] .
\end{aligned}
$$

Hence

$\int_{\substack{1 / 2 \varepsilon \leq s \leq t \\(r, s) \in D}}|\widetilde{Q}| d r d s \leq c t e J_{1} \varepsilon^{3 / 2}, \quad J(t) \leq J\left(\frac{1}{2 \varepsilon}\right)+\int_{\substack{1 / 2 \varepsilon \leq s \leq t \\(r, s) \in D}}|\widetilde{Q}| d r d s \leq \frac{1}{2} J_{1} \varepsilon$.

In order to estimate $M(t)$, we note that

$$
\left\{\begin{array}{l}
\partial_{t} A+(\alpha-c) \partial_{r} A=-2 \rho c Z_{1}+\frac{A B}{r^{2}}-\frac{\rho B}{r} \\
\partial_{t} B+(\alpha-c) \partial_{r} B=-2 c^{2} Z_{1}+\frac{B^{2}}{r^{2}}+\frac{c^{2}(\rho)}{\rho} \frac{A}{r}
\end{array}\right.
$$


Then, for $\varepsilon$ small enough, we have

$$
(|A|+|B|)(r, t) \leq \frac{3}{4} M_{1} \varepsilon
$$

Similarly, $V(t) \leq V_{1} \varepsilon^{1 / 2} / 2$.

Therefore, by continuous induction, we know that Lemma 3 holds.

Along $\Gamma_{\sigma_{0}}^{+} \subset D$, we define $w(t)=-Z_{1}(r(t), t)$. It is easy to know that $w$ satisfies the equation:

$$
w^{\prime}(t)=a_{0}(t) w^{2}+a_{1}(t) w+a_{2}(t)
$$

where

$$
\begin{aligned}
a_{0}(t)= & \left(\frac{\rho c^{\prime}+c}{r}\right)(r(t), t), \\
a_{1}(t)= & \frac{Z_{2}}{r}\left(3 c^{\prime} \rho+\frac{B}{r}\right)+\frac{1}{r^{2}}\left[3 B+\frac{c A}{\rho}+\frac{c^{\prime} A}{2}+\frac{c^{\prime} B \rho}{2 c}+\left(\frac{c^{2}(\rho)}{\rho}\right)^{\prime} \frac{\rho A}{c}\right. \\
& \left.\quad-\frac{B \bar{\rho}}{2 \rho}-\frac{A B}{2 \rho r}+\frac{c r}{2 \rho}(\rho-\bar{\rho})\right], \\
a_{2}(t)=- & \frac{Z_{2}}{r}\left(c-\frac{B}{r}\right)-\frac{Z_{2}^{2}}{r}\left[B-\frac{c A}{\rho}\left(\frac{c^{2}(\rho)}{\rho}\right)^{\prime} \frac{\rho A}{c}+\frac{\bar{c} B \rho}{2 c}+\frac{c A}{\rho}+\frac{c^{\prime} A}{2}\right. \\
& \left.+\frac{B \rho}{2 \rho}-\frac{B c}{2 \rho r}-\frac{c r}{2}-\frac{\bar{\rho} c r}{2 \rho}\right]-\frac{1}{r^{2}}\left(-\frac{c A}{2 \rho}+B-\frac{\bar{\rho} B}{2 \rho}\right) \\
& -\frac{1}{r^{3}}\left[-\frac{B^{2}}{c}-\left(\frac{c^{2}(\rho)}{\rho}\right)^{\prime} \frac{A^{2}}{2 c}-\frac{3 A B}{2 \rho}+\frac{\bar{\rho} B c}{2 \rho^{2}}\right]-\frac{c A B}{2 \rho^{2} r^{4}} .
\end{aligned}
$$

Lemma 3 and the above calculation give out the following estimates

$$
a_{0}(t)=\frac{\bar{\rho} c^{\prime}(\bar{\rho})+\bar{c}}{\bar{c} t}+O\left(t^{-2}\right), \quad\left|a_{1}(t)\right| \leq c t e \frac{\varepsilon^{1 / 2}}{t^{2}}, \quad\left|a_{2}(t)\right| \leq c t e \frac{\varepsilon^{1 / 2}}{t^{2}}
$$

and

$$
\begin{aligned}
w\left(\frac{1}{2 \varepsilon}\right)=- & \frac{1}{2}\left[\frac{\left(\rho_{a}-\bar{\rho}\right)\left(r\left(\frac{1}{2 \varepsilon}\right), \frac{1}{2 \varepsilon}\right)+r\left(\frac{1}{2 \varepsilon}\right) \partial_{r} \rho_{a}\left(r\left(\frac{1}{2 \varepsilon}\right), \frac{1}{2 \varepsilon}\right)}{\rho_{a}\left(r\left(\frac{1}{2 \varepsilon}\right), \frac{1}{2 \varepsilon}\right)}\right. \\
& \left.+\frac{\alpha_{a}\left(r\left(\frac{1}{2 \varepsilon}\right), \frac{1}{2 \varepsilon}\right)+r\left(\frac{1}{2 \varepsilon}\right) \partial_{r} \alpha_{a}\left(r\left(\frac{1}{2 \varepsilon}\right), \frac{1}{2 \varepsilon}\right)}{c\left(\rho_{a}\left(r\left(\frac{1}{2 \varepsilon}\right), \frac{1}{2 \varepsilon}\right)\right)}\right]+o(\varepsilon) .
\end{aligned}
$$


As $\varepsilon$ is small enough, from the proof in Lemma 3, we know $r(1 / 2 \varepsilon) \geq$ $(\bar{c} / 2)(1 / 2 \varepsilon)$. So it turns out:

$$
\left|\partial_{x, t}^{\beta}\left(\omega_{0}-\frac{F_{0}(r-\bar{c} t)}{r}\right)\right|_{r=r(1 / 2 \varepsilon), t=1 / 2 \varepsilon} \leq \frac{c t e}{\left(1+\frac{1}{2 \varepsilon}\right)^{2}} \leq c_{t e \varepsilon^{2}},|\beta| \geq 0
$$

and

$$
\begin{gathered}
\alpha_{a}\left(r\left(\frac{1}{2 \varepsilon}\right), \frac{1}{2 \varepsilon}\right)=\varepsilon\left[\frac{\partial_{q} F_{0}\left(r\left(\frac{1}{2 \varepsilon}\right)-\frac{\bar{c}}{2 \varepsilon}\right)}{r\left(\frac{1}{2 \varepsilon}\right)}-\frac{F_{0}\left(r\left(\frac{1}{2 \varepsilon}\right)-\frac{\bar{c}}{2 \varepsilon}\right)}{r^{2}\left(\frac{1}{2 \varepsilon}\right)}\right]+o(\varepsilon), \\
\rho_{a}-\bar{\rho}=\frac{\varepsilon \bar{\rho}}{\bar{c}} \frac{\partial_{q} F_{0}\left(r\left(\frac{1}{2 \varepsilon}\right)-\frac{\bar{c}}{2 \varepsilon}\right)}{r\left(\frac{1}{2 \varepsilon}\right)}-\frac{\varepsilon \bar{\rho}}{\bar{c}} r\left(\frac{1}{2 \varepsilon}\right)\left[-\frac{\partial_{q}^{2} F_{0}\left(r\left(\frac{1}{2 \varepsilon}\right)-\frac{\bar{c}}{2 \varepsilon}\right)}{r\left(\frac{1}{2 \varepsilon}\right)}\right. \\
\left.+\frac{\partial_{q} F_{0}\left(r\left(\frac{1}{2 \varepsilon}\right)-\frac{\bar{c}}{2 \varepsilon}\right)}{r^{2}\left(\frac{1}{2 \varepsilon}\right)}\right]+o(\varepsilon) .
\end{gathered}
$$

Substituting them into (12), we obtain

$$
w\left(\frac{1}{2 \varepsilon}\right)=-\frac{\varepsilon \partial_{q}^{2} F_{0}\left(r\left(\frac{1}{2 \varepsilon}\right)-\frac{\bar{c}}{2 \varepsilon}\right)}{\bar{c}}+o(\varepsilon) .
$$

By $(10),\left|r(1 / 2 \varepsilon)-\bar{c} / 2 \varepsilon-\sigma_{0}\right| \leq c t e \varepsilon \ln (1+1 / 2 \varepsilon)$, then

$$
w\left(\frac{1}{2 \varepsilon}\right)=-\frac{\varepsilon}{2}\left[\sigma_{0}^{2} \partial_{q} v_{1}^{0}\left(\sigma_{0}\right)+3 \sigma_{0} v_{1}^{0}\left(\sigma_{0}\right)+\frac{\bar{c}}{\bar{\rho}}\left(\sigma_{0} \partial_{q} \rho_{0}\left(\sigma_{0}\right)+\rho_{0}\left(\sigma_{0}\right)\right)\right]+o(\varepsilon)
$$

Denote $K=\left(\int_{1 / 2 \varepsilon}^{T}\left|a_{2}(t)\right| d t\right) \exp \left(\int_{1 / 2 \varepsilon}^{T}\left|a_{1}(t)\right| d t\right)$, and note the equation (11) satisfies the conditions of [2, Lemma 1.3.2.] in $1 / 2 \varepsilon \leq t \leq T$, so we obtain:

$$
\left(\int_{1 / 2 \varepsilon}^{T} a_{0}(t) d t\right) \exp \left(-\int_{1 / 2 \varepsilon}^{T}\left|a_{1}(t)\right| d t\right)<\left(w\left(\frac{1}{2 \varepsilon}\right)-K\right)^{-1}
$$

that is

$$
\begin{aligned}
& \frac{\bar{\rho} c^{\prime}(\bar{\rho})+\bar{c}}{\bar{c}}(\ln T+\ln 2 \varepsilon+O(\varepsilon)) e^{-c t e \varepsilon^{3 / 2}} \\
& <\left[-\frac{\varepsilon}{2}\left(\sigma_{0}^{2} \partial_{q} v_{1}^{0}\left(\sigma_{0}\right)+3 \sigma_{0} v_{1}^{0}\left(\sigma_{0}\right)+\frac{\bar{c}}{\bar{\rho}} \sigma_{0} \partial_{q} \rho_{0}\left(\sigma_{0}\right)+\frac{\bar{c}}{\bar{\rho}} \rho_{0}\left(\sigma_{0}\right)\right)\right. \\
& \left.\quad+o(\varepsilon)+\text { cte }^{3 / 2}\right]^{-1} .
\end{aligned}
$$

Let $\varepsilon \rightarrow 0$, we get

$$
\lim _{\varepsilon \rightarrow 0} \varepsilon \ln T_{\varepsilon} \leq \tau_{0} .
$$

Combine (13) with the conclusion in $\S 2$, we know Theorem holds. 


\section{REFERENCES}

[1] S. Klainerman, Uniform decay estimate and the Lorentz invariance of the classical wave equation, Comm. Pure Appl. math., 38 (1985), 321-332.

[2] L. Hömander, Nonlinear hyperbolic differential equations, Lectures, 1986-1987.

[3] T. Sideris, Formation of solution singularities in three dimensional compressible fluids, Comm. Math. Phys., 101 (1985), 475-487.

[4] S. Alinhac, Temps de vie des solutions regulieres de equations d'Euler compressibles axisymetriques en dimendion deux, Invent. Math., 111 (1993), 627-667.

[5] R. Courant, K. O. Friedrichs, Supersonic flow and shock waves, Wiley Interscience, New York, 1949.

[6] L. Hömander, The lifespan of classical solutions of nonlinear hyperbolic equations, Mittag-Leffler report No. 5 (1985).

[7] F. John, Blow-up of radial solutions of $u_{t t}=c^{2}\left(u_{t}\right) \Delta u$ in three space dimensions, Mat. Apl. Comput., 4 (1985), No. 1, 3-18.

[8] S. Klainerman, Remarks on the global Sobelev inequalities in the Minkowski space $R^{n+1}$, Comm. Pure Appl. Math., 40 (1987), 111-117.

Huicheng Yin

Department of Mathematics

Nanjing University

Nanjing 210093

P. R. China

Qingjiu Qiu

Department of Mathematics

Nanjing University

Nanjing 210093

P. R. China 